\title{
NORMALIZED FINITE FRACTIONAL DIFFERENCES: COMPUTATIONAL AND ACCURACY BREAKTHROUGHS
}

\author{
RAFAŁ STANISŁAWSKI，KRZYSZTOF J. LATAWIEC \\ Institute of Control and Computer Engineering \\ Opole University of Technology, ul. Sosnkowskiego 31, 45-272 Opole, Poland \\ e-mail: \{r.stanislawski,k.latawiec\}@po.opole.pl
}

\begin{abstract}
This paper presents a series of new results in finite and infinite-memory modeling of discrete-time fractional differences. The introduced normalized finite fractional difference is shown to properly approximate its fractional difference original, in particular in terms of the steady-state properties. A stability analysis is also presented and a recursive computation algorithm is offered for finite fractional differences. A thorough analysis of computational and accuracy aspects is culminated with the introduction of a perfect finite fractional difference and, in particular, a powerful adaptive finite fractional difference, whose excellent performance is illustrated in simulation examples.
\end{abstract}

Keywords: fractional difference, Grünwald-Letnikov difference, stability analysis, recursive computation, adaptive systems.

\section{List of abbreviations}

AFFD Adaptive Finite Fractional Difference

FD Fractional Difference

FFD Finite Fractional Difference

FIR Finite Impulse Response

IIR Infinite Impulse Response

LS Least Squares

NFFD Normalized Finite Fractional Difference

OBF Orthonormal Basis Functions

PFFD Perfect Finite Fractional Difference

\section{Introduction}

Non-integer or fractional-order dynamic models have recently attracted considerable research interest. Their specific properties can make them more adequate in the modeling of selected industrial systems (Riu et al., 2001; Zaborowsky and Meylaov, 2001; Petráš and Vinagre, 2002; Delavari et al., 2010). A number of discrete-time fractional difference systems have been modeled via both a transfer function or difference equation models (Lubich,
1986; Ortigueira, 2000; Ostalczyk, 2000; Petráš et al., 2000) and state space ones (Sierociuk and Dzieliński, 2006; Dzieliński and Sierociuk, 2008; Kaczorek, 2008).

Various approximations to fractional differences have been pursued. Since an FD represents in fact (a sort of) an infinite impulse response filter, one solution has been to least-squares fit an impulse/step response of a discrete-time integer-difference IIR filter to that of the associated FD (Vinagre et al., 2000; Chen et al., 2003; Barbosa and Machado, 2006). The problem here is to propose a "good" structure of the integer-difference filter, possibly involving a low number of parameters. On the other hand, an LS fit of the FIR filter to FD has been analyzed in the frequency domain (Tseng et al., 2000), with the high-order optimal filter providing a good approximation accuracy, at the cost of a remarkable computational effort, though. Another approach behind that research direction has been the employment of an approximating filter incorporating orthonormal basis functions (Maione, 2006). That promising solution has, however, suffered from the necessity to select dominant OBF pole(s), which may significantly affect the approximation accuracy. It is finally worth mentioning about yet another, numerical approach to the approximation of fractional systems in the context of integral equations (Momani and Odibat, 2007; Bandrowski et al., 2010; Saeedi et al., 2011), the approach 
suffering, again, from a computational burden.

Apart from the computational aspects to be covered later on, our reservation against the above approximation approaches results form the fact that the proposed approximating IIR/FIR/OBF filters are quite arbitrary in that they do not use any a priori knowledge about the mathematical (not to say physical) structure of FD. Therefore we advocate an alternative approach relying on the approximation of the FD filter with its truncated, finite-memory version (Podlubny, 1999; Dzieliński and Sierociuk, 2008; Monje et al., 2010). In analogy to finite impulse response the term finite FD, or FFD, has been coined (Stanisławski, 2009). FFD may, however, suffer from (remarkable) steady-state errors as compared to FD. To cope with this, we have introduced what is here referred to as normalized FFD, or NFFD (Stanisławski and Latawiec, 2010; 2011). This paper presents a number of new results concerning an efficient approximation of FD by (various versions of) NFFD. In particular, the results concern the steady-state error analysis, (preliminary) stability analysis, recursive computation, variable-order and robustness issues for NFFD modeling.

Having introduced the FD modeling problem, the Grünwald-Letnikov fractional discrete-time difference is recalled, together with its NFFD approximation, in Section 2. That section also includes the steady-state error analysis for NFFD, thus resulting in the introduction of a specific normalizing factor whose properties are summarized in a series of lemmas and theorems. Finally, Section 2 provides tools for recursive computation of NFFD, which appears an order faster than its off-line counterpart. Variable-order NFFD is covered in Section 3. In particular, a recursive computation algorithm provides a linear time complexity design. An application of NFFD in modeling of state-space systems is presented in Section 4, with stability and steady-state accuracy results yielded. In Section 5, the results are illustrated in the first series of simulations, which show the need for improvement of NFFD. Accordingly, NFFD is modified in Section 6, yielding a sort of an adaptive version of NFFD, or AFFD. Finally, another new modification of NFFD, called perfect FFD, or PFFD, is introduced in Section 7. The two modifications, that is, AFFD and PFFD, are presented in the variable-order framework in Section 8, with the superiority of AFFD indicated. The second series of simulation examples of Section 10 confirms the theoretical yieldings of Sections 6 to 9. Conclusions of Section 11 summarize the achievements of the paper.

\section{Fractional discrete-time difference}

A simple generalization of the familiar Grünwald-Letnikov difference (Miller and Ross, 1993) is the fractional difference in discrete time $t$, described by the equation (Oldham and Spanier, 1974; Sierociuk and Dzieliński, 2006; Kaczorek, 2008; Guermah et al., 2010)

$$
\begin{aligned}
\Delta^{\alpha} x(t) & =\sum_{j=0}^{t} P_{j}(\alpha) x(t) q^{-j} \\
& =x(t)+\sum_{j=1}^{t} P_{j}(\alpha) x(t) q^{-j}, \quad t=0,1, \ldots,
\end{aligned}
$$

where $\alpha \in(0,2)$ is the fractional order, $q^{-1}$ is the backward shift operator and

$$
P_{j}(\alpha)=(-1)^{j} C_{j}(\alpha),
$$

with

$C_{j}(\alpha)=\left(\begin{array}{c}\alpha \\ j\end{array}\right)= \begin{cases}1, & j=0, \\ \frac{\alpha(\alpha-1) \ldots(\alpha-j+1)}{j !}, & j>0 .\end{cases}$

Remark 1. It is well known that $\sum_{j=0}^{\infty} P_{j}(\alpha)=0$ or, equivalently, $\sum_{j=1}^{\infty} P_{j}(\alpha)=-1$.

Remark 2. Possible accounting for the sampling period $T$ when transferring from a continuous-time derivative to the discrete-time difference results in dividing the right-hand side of Eqn. (1) by $T^{\alpha}$ (Monje et al., 2010). Operating without $T^{\alpha}$ as in the sequel corresponds to putting $T=1$ or to the substitution of $P_{j}(\alpha)$ for $P_{j}(\alpha) / T^{\alpha}, j=0, \ldots, t$.

Note that each element in Eqn. (1) from time $t$ back to 0 is non-zero, so that each incoming sample of the signal $x(t)$ increases the complication of the model equation. In the limit, as $t \rightarrow+\infty$, we end up with computational explosion.

2.1. Finite fractional difference. Stanisławski (2009) considered truncated or finite fractional differences (in analogy to FIR) for practical, feasibility reasons, with the convergence to zero of the series $C_{j}(\alpha)$ enabling to assume $C_{j}(\alpha) \approx 0$ for some $j>\bar{J}$, where $\bar{J}$ is the number of backward signal samples used to calculate the fractional difference. We will further proceed with FFD, to be formally defined below.

Definition 1. Let the fractional difference be defined as in Eqns. (1)-(3). Then the finite fractional difference is defined as

$$
\Delta^{\alpha} x(t, J)=x(t)+\sum_{j=1}^{J} P_{j}(\alpha) x(t) q^{-j},
$$

where $J=\min (t, \bar{J})$ and $\bar{J}$ is the upper bound to $j$ when $t>\bar{J}$. 
FFD has been analyzed in some papers under the heading of a practical implementation of FD (Kaczorek, 2008; Busłowicz and Kaczorek, 2009), or a finite difference (Dzieliński and Sierociuk, 2008; Monje et al., 2010) or a short-memory difference (Podlubny, 1999).

An important problem encountered in FFD-based modeling is an incorrect steady-state gain of the model, with its discrepancy with respect to the corresponding FD one being dependent on $\bar{J}$. This can be illustrated in the step signal $x(t)=x_{0} \mathbf{1}(t)$ whose FFD with $\alpha=0.5$ and $\bar{J}=20$ produces $\left.\lim _{t \rightarrow \infty} \Delta^{\alpha} x(t, J)\right|_{x(t, J)=x_{0} \mathbf{1}(t)} \approx$ $0.12 x_{0}$, whereas the limit is zero for FD, that is,

$$
\left.\lim _{t \rightarrow \infty} \Delta^{\alpha} x(t)\right|_{x(t)=x_{0} \mathbf{1}(t)}=0 .
$$

This may cause a (considerable) difference between steady-state outputs of a plant and its FFD model. Also, this may affect a stability condition for FFD. Therefore, some special means must be applied to provide steady-state error-free FFD modeling with reasonably low $\bar{J}$.

2.2. Normalized finite fractional difference. Here we propose an effective tool to cope with the problem of a steady-state error in FFD modeling. In order to provide $\left.\lim _{t \rightarrow \infty} \Delta^{\alpha} x(t, J)\right|_{x(t, J)=x_{0} \mathbf{1}(t)}=0$, it its sufficient to incorporate a normalization factor $N=N(\bar{J})$ into FFD to obtain normalized FFD.

Definition 2. Let the fractional difference be defined as in Eqns. (11) - (3) and the finite fractional difference be defined as in Definition 1 . Then the normalized finite fractional difference is defined as

$$
\Delta_{N}^{\alpha} x(t, J)=x(t)+\frac{1}{N} \sum_{j=1}^{J} P_{j}(\alpha) x(t) q^{-j},
$$

where $N=N(\bar{J})$ is the normalizing factor.

Lemma 1. Let NFFD be defined as in Definition 2 Then the steady-state error-free modeling of FD with Eqn. (6) can be provided by the selection

$$
N=-\sum_{j=1}^{\bar{J}} P_{j}(\alpha)
$$

Proof. We assume that the steady state $x_{s s}$ for NFFD is reached for a sufficiently high $t$, that is, $t>\bar{J}$. In order to satisfy the steady-state error-free condition, one must have (cf. Eqn. (5))

$$
\begin{aligned}
& \left.\lim _{t \rightarrow \infty} \Delta_{N}^{\alpha} x(t, J)\right|_{x(t, J)=x_{s s}} \\
& =x_{s s}+\frac{1}{N} \sum_{j=1}^{\bar{J}} P_{j}(\alpha) x_{s s}=0 .
\end{aligned}
$$

This ends up with Eqn. (7).
Lemma 2. Let $N=N(\bar{J})$ as in Eqn. (7). Then

$$
\lim _{\bar{J} \rightarrow \infty} N(\bar{J})=1 \text {. }
$$

Proof. It follows immediately from Remark 1 and Eqn. (7).

The asymptotic properties of NFFD as $t \rightarrow \infty$ and $J \rightarrow \infty$ are summarized in the following two theorems.

Theorem 1. Let $N=N(\bar{J})$ be selected as in Eqn. (7). Then

$$
\lim _{t \rightarrow \infty} \Delta_{N}^{\alpha} x(t, J)=\lim _{t \rightarrow \infty} \Delta^{\alpha} x(t)
$$

Proof. It follows immediately from Definition 2 and Lemma 1.

Theorem 2. Let $N=N(\bar{J})$ be selected as in Eqn. (7). Then

$$
\lim _{\bar{J} \rightarrow \infty} \Delta_{N}^{\alpha} x(t, J)=\Delta^{\alpha} x(t)
$$

Proof. It follows immediately from Definition 2 and Lemma2.

We will jointly describe FD, FFD and NFFD as

$$
\Delta_{N}^{\alpha} x(t, J)=x(t)+X(t, J)
$$

with

$$
X(t, J)=\frac{1}{N} \sum_{j=1}^{J} P_{j}(\alpha) x(t) q^{-j}
$$

and $\bar{J} \rightarrow \infty$ (implying $N \rightarrow 1$ ) for FD, $N=1$ for FFD and $N=N(\bar{J})$ as in Eqn. (7) for NFFD.

The NFFD as in Eqn. (6) with the normalizing factor as in Eqn. (7) will sometimes be referred to as an "off-line NFFD".

2.3. Recursive computation of NFFD. Essential computational savings can be obtained when computing (some elements of) finite fractional differences in a recursive way.

We consider an NFFD with the normalizing factor $N=N(\bar{J})$ selected as in Lemma 1, with $J$ substituted for $\bar{J}$,

$$
\Delta_{N}^{\alpha} x(t, J)=x(t)+X(t, J), \quad \forall t=0,1, \ldots,
$$

where

$$
X(t, J)=\frac{1}{N(J)} B(t, J)
$$


with $N(J)=-\sum_{j=1}^{J} P_{j}(\alpha), P_{j}(\alpha)$ defined in Eqns. (1) and (2) and $B(t, J)=\sum_{j=1}^{J} P_{j}(\alpha) x(t-j)$. Then $N(J)$ in Eqn. 14 can be computed recursively as

$$
\begin{aligned}
P_{J}(\alpha) & =\left(1-\frac{\alpha+1}{J}\right) P_{J-1}(\alpha), \\
N(J) & =N(J-1)-P_{J}(\alpha),
\end{aligned}
$$

with $P_{0}(\alpha)=1, N(0)=0$ and $J=1,2, \ldots, \bar{J}$.

In fact, Eqns. 15 and 16 can be immediately obtained if we note that

$$
P_{j}(\alpha)=(-1)^{J-1} C_{J-1}(\alpha)\left(1-\frac{\alpha+1}{J}\right)
$$

and

$$
N(J)=-\sum_{j=1}^{J-1} P_{j}(\alpha)-P_{J}(\alpha) .
$$

Remark 3. Observe that each recursion (15) and (16) with respect to $J$ is executed only once at time $t=J=$ $1, \ldots, \bar{J}$.

Remark 4. Note that the recursive computation of NFFD is started (at $J=1$ ) from the regular difference $\Delta x(t)=$ $x(t)-x(t-1)$ and it converges to the off-line computed NFFD for $J=\bar{J}$.

The recursive NFFD will sometimes be referred to as an "on-line NFFD".

\subsubsection{Computational efforts for off-line vs. recursive} NFFDs. Time complexity $\mathcal{T}_{\text {off }}(\bar{J})$ for one step of the simulation process for off-line NFFD can be easily shown to be equal to

$$
\mathcal{T}_{\text {off }}(\bar{J})=2 \bar{J}^{2}-\frac{1}{2} \bar{J}+1 \in \mathcal{O}\left(\bar{J}^{2}\right) .
$$

In contrast, time complexity $\mathcal{T}_{\text {on }}(\bar{J})$ for one step of the simulation process for on-line NFFD is as low as

$$
\mathcal{T}_{\text {on }}(\bar{J})=2 \bar{J}+6 \in \mathcal{O}(\bar{J})
$$

Clearly, the on-line NFFD algorithm runs in the order of $\bar{J}$, which is an order faster than its off-line counterpart.

\section{Variable-order NFFD}

Variable-order fractional differences, or fractional differences with time-varying order $\alpha_{t}=\alpha(t)$, were studied by Ostalczyk (2010), or, more generally, by Sun et al. (2009), as well as Valério and Sá da Costa (2011), with a high computational burden experienced. Here we present a computationally effective, recursive algorithm for NFFD with a time-varying (state-independent) order.
Remark 5. It is interesting to note that Lemmas 1 and 2 as well as Theorems 11 and 2 are still valid for a time-varying order $\alpha_{t}$. This results from the fact that $\lim _{j \rightarrow \infty} P_{j}\left(\alpha_{t}\right)=0$.

We consider an NFFD with a time-varying order $\alpha_{t}=\alpha(t)$ and the normalizing factor selected as in Lemma 1 with $J$ substituted for $\bar{J}$,

$$
\Delta_{N}^{\alpha_{t}} x(t, J)=x(t)+X(t, J), \quad \forall t=0,1, \ldots,
$$

where

$$
X(t, J)=\frac{1}{N_{t}(J)} B(t, J)
$$

with $N_{t}(J)=-\sum_{j=1}^{J} P_{j}\left(\alpha_{t}\right), P_{j}\left(\alpha_{t}\right)$ being defined in Eqns. (1) and (2) (with $\alpha_{t}$ substituted for $\alpha$ ), and $B(t, J)=\sum_{j=1}^{J} P_{j}\left(\alpha_{t}\right) x(t-j)$. Then $N_{t}(J)$ in Eqn. (20) can be computed recursively as

$$
\begin{aligned}
& P_{K}\left(\alpha_{t}\right)=\left(1-\frac{\alpha_{t}+1}{K}\right) P_{K-1}\left(\alpha_{t}\right), \\
& N_{t}(K)=N_{t}(K-1)-P_{K}\left(\alpha_{t}\right),
\end{aligned}
$$

$\forall K=1, \ldots, J$, with $P_{0}\left(\alpha_{t}\right)=1, N_{t}(0)=0$ and $J$ defined as in Definition 1 .

Equations (21) and (22) are the extensions of Eqns. (15) and (16), respectively, with the reindexing with $K$ being necessary due to the time-varying $\alpha_{t}$.

Remark 6. Note that the recursions 21) and 221 are now calculated $J$ times at each time instant $t=0,1, \ldots$.

3.1. Computational effort revisited: Part 1. Time complexity $\mathcal{T}_{\text {var }}(\bar{J})$ for one step of the simulation process for recursive NFFD with a time-varying order is now

$$
\mathcal{T}_{\text {var }}(\bar{J})=6 \bar{J}+1 \in \mathcal{O}(\bar{J}) .
$$

Again, the linear time algorithm is thus obtained.

3.2. Round-off errors. It is well known that long-term recursive computations may be affected with propagation of computer round-off errors (Verhaegen, 1989; Liavas and Regalia, 1999). A simple remedy is to occasionally reset the recursive algorithm, while maintaining an old "frozen" model until a new stationary state of the restarted algorithm has been reached. However, in the long run, FD/NFFD models are becoming "saturated", even for a time-varying order (cf. Remark 5), so that long-term (steady-state) computations for such models cannot take us any further. In fact, the (steady-state) gain of such models is independent of the order $\alpha$. 


\section{State space model description}

Consider a discrete-time state space system described by the (constant-order) fractional model

$$
\begin{aligned}
\Delta^{\alpha} x(t+1) & =A_{d} x(t)+B u(t), \quad x(0)=x_{0}, \\
y(t) & =C x(t)+D u(t),
\end{aligned}
$$

where $x(t) \in \mathbb{R}^{n}, u(t) \in \mathbb{R}^{n_{u}}$ and $y(t) \in \mathbb{R}^{n_{y}}$ are the state, input and output vectors, respectively, $A_{d} \in \mathbb{R}^{n \times n}$, $B \in \mathbb{R}^{n \times n_{u}}, C \in \mathbb{R}^{n_{y} \times n}$ and $D \in \mathbb{R}^{n_{y} \times n_{u}}$. Without loss of generality we will assume in the sequel that the initial vector $x_{0}$ is zero, the more so that we will operate on finite-memory FD approximations that do not trace back to $x_{0}$.

Accounting that the FD is calculated by the formula (1), Eqn. (24) can be presented in the following form (cf. Monje et al., 2010):

$$
\begin{aligned}
x(t+1)= & \left(A_{d}+\alpha I\right) x(t) \\
& -\sum_{j=2}^{t+1} P_{j}(\alpha) x(t-j+1)+B u(t) .
\end{aligned}
$$

Using Definitions 1 and 2, we can jointly present FD/FFD/NFFD-based discrete-time state equations as

$$
\begin{aligned}
x(t+1)= & \left(A_{d}+\frac{\alpha}{N} I\right) x(t) \\
& -\frac{1}{N} \sum_{j=2}^{J} P_{j}(\alpha) x(t-j+1)+B u(t),
\end{aligned}
$$

with $J$ and $N=N(\bar{J})$ defined as in Definitions 1 and 2, respectively.

Note that Eqn. 27) can be regarded as the most general fractional-difference state equation (within the fractional-difference class considered here) including the NFFD-based one, FFD-based one (for $N=1$ ) and FD-based one (for $\bar{J} \rightarrow \infty$ implying $N \rightarrow 1$ ).

Remark 7. Possible accounting for the sampling period $T$ (when transferring from a continuous-time derivative to the discrete-time difference) results in the substitutions $A_{d} \rightarrow A_{d} T^{\alpha}$ and $B \rightarrow B T^{\alpha}$ in Eqns. 26) and 27) (Monje et al., 2010).

4.1. Stability. Our simple NFFD stability result is now offered as follows

Theorem 3. The NFFD-based discrete-time state equation (27), with $\alpha \in(0,2)$ and $N$ selected as in Lemma 1 is asymptotically stable if

$$
\|\underline{A}\|<\phi(\alpha),
$$

where $\|\cdot\|$ is a matrix norm, $\underline{A}=A_{d}+\frac{\alpha}{N} I, \phi(\alpha)=\alpha / N$ for $\alpha \in(0,1)$ and $\phi(\alpha)=2-\alpha / N$ for $\alpha \in(1,2)$.
Proof. See Appendix A.

Interestingly, the stability condition (28) for the NFFD-based state space system with the normalizing factor selected according to Lemma 1 includes that for the FD-based one (cf. Monje et al., 2010), in which case $\bar{J} \rightarrow \infty$ implying $N \rightarrow 1$, and that for the FFD-based one $(N=1)$ as well as that for the "regular" difference $(\alpha=1$ and $N=1)$.

Remark 8. For the FFD-based system, the result of Theorem 3 is similar to those of Dzieliński and Sierociuk (2008) as well as Monje et al. (2010). More general stability results for the FFD-based system were given by Guermah et al. (2010), as well as Stojanovic and Debeljkovic (2010).

\subsection{Steady-state accuracy.}

Theorem 4. Let the steady-state output error for the FFD/NFFD-based state-space model with respect to the FD one be defined as

$$
\epsilon_{s s}=\lim _{t \rightarrow \infty}\left\{\epsilon_{y}(t)=y_{N F D}(t)-y_{F D}(t)\right\},
$$

where $y_{N F D}(t)$ and $y_{F D}(t)$ are the outputs of the FFD/NFFD-based and FD-based state-space models, respectively. Then

$$
\epsilon_{s s}=C\left(F-A_{d}\right)^{-1} F A_{d}^{-1} B u_{s s},
$$

where $u_{s s}$ is the steady state input and

$$
F=\left[1+\frac{1}{N} \sum_{j=1}^{\bar{J}} P_{j}(\alpha)\right] I,
$$

with $N=N(\bar{J})$ as in Lemma 1 for the NFFD-based system and $N=1$ for the FFD-based one.

Proof. See Appendix B.

It is clear now that seady-state error-free output modeling can be obtained for the NFFD-based system only. In fact, the matrix $F$ is zero in that case.

4.3. Variable-order state space model. For the NFFD-based state space model with time-varying (state-independent) order $\alpha_{t}, t=0,1, \ldots$, we have the following two interesting remarks.

Remark 9. Unlike FD-based and NFFD-based systems, the FFD-based state-space model has no steady state for a time-varying order $\alpha_{t}$. This results immediately from Theorem 4 In fact, the matrix $F$ is time-varying in that case.

Remark 10. The NFFD steady-state error-free modeling result of Theorem 4 is still valid for time-varying $\alpha_{t}$; in fact, the matrix $F$ is zero, even for time-varying $\alpha_{t}$. 


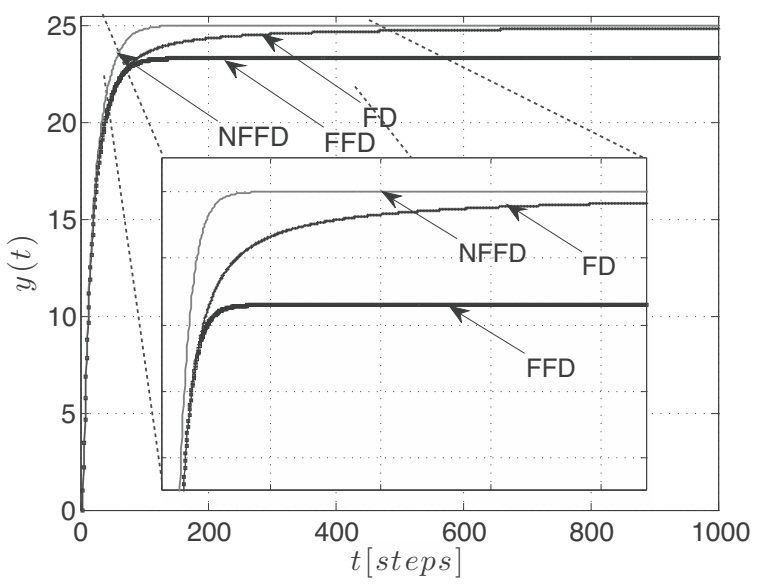

Fig. 1. Time plots of outputs for FD, FFD and NFFD-based state-space systems: Example 1

\section{Simulation examples: Part 1}

NFFD with $N(\bar{J})$ selected as in Lemma1 has been shown to provide a good approximation accuracy with respect to FD for high $\bar{J}$ and, in particular, for values of $\alpha$ being close to unity (Stanisławski and Latawiec, 2011). Here we demonstrate a rather poor modeling performance in case we use NFFD for low values of $\bar{J}$ and, in particular, low values of $\alpha$. This will trigger the NFFD modification process.

Example 1. Consider an FD-based discrete-time state space system with

$$
\begin{gathered}
A_{d}=\left[\begin{array}{cc}
-0.1 & 0 \\
1 & -0.4
\end{array}\right], \quad B=\left[\begin{array}{l}
1 \\
0
\end{array}\right], \\
C=\left[\begin{array}{ll}
0 & 1
\end{array}\right], \quad D=[0], \quad \alpha=0.85
\end{gathered}
$$

and its FFD-based state-space model. The steady-state output values of the model for $u=1(t)$ and various values of $\bar{J}$ are presented in Table 1 Additionally, the outputs for FFD and NFFD (with $\bar{J}=50$ ) vs. FD-based systems are plotted in Fig. 1 .

Table 1. Steady-state output of the FFD-based system.

\begin{tabular}{|c||c|c|c|c|c|c|}
\hline $\bar{J}$ & 10 & 50 & 100 & 500 & 1000 & $\infty$ \\
\hline$y(\infty)$ & 19.31 & 23.30 & 24.03 & 24.75 & 24.85 & 25 \\
\hline
\end{tabular}

Example 2. Consider an NFFD as in (6), with $N$ selected according to Lemma 1 and $\alpha=0.6, \bar{J}=40$. Figure 2 presents the convergence process of $\underline{X}(t, J)=-X(t, J)$ for the recursive version of NFFD to that for the off-line version.

Note that we choose to plot $\underline{X}(t, J)$ here as the (positive) values of that signal firmly illustrate the behavior of the most important component of FFD/NFFD, that is, the second component on the right-hand sides of Eqns. (4) and (6).

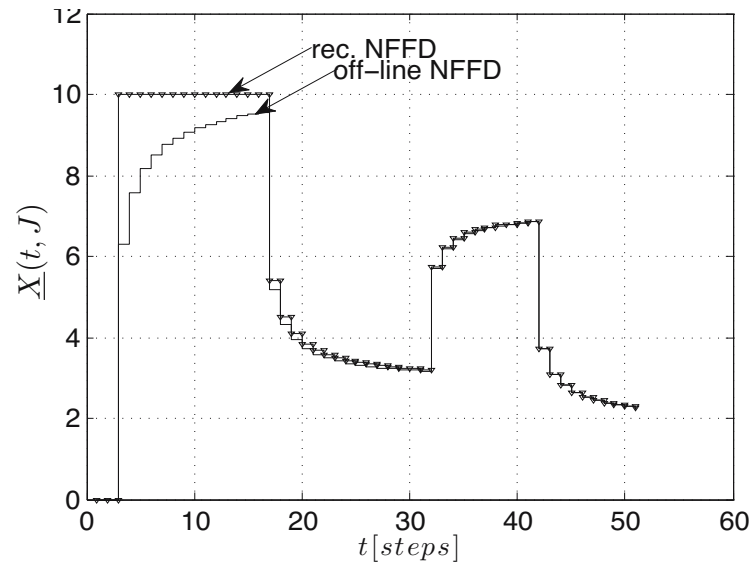

Fig. 2. Time plots of $\underline{X}(t, J)$ for the recursive and off-line versions of the NFFD model: Example 2.

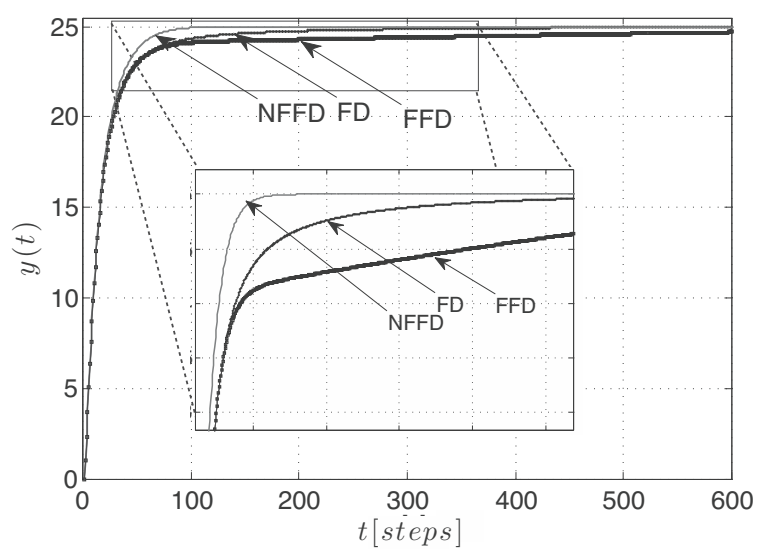

Fig. 3. Output time plots for varying-order FD vs. FFD and (offline) NFFD-based state-space systems: Example 3.

Example 3. Consider a varying-order state space model with the elements $A_{d}, B, C, D$ as in Example $1 \bar{J}=50$ and

$$
\alpha_{t}=0.9+0.0001 t, \quad t \in(0,600) .
$$

Time plots of outputs for FD, FFD and NFFD-based systems (Fig. 3) confirm our theoretical results summarized in Remarks 9 and 10.

We have also frequency-domain analyzed the behavior of (constant-order) FD, FFD and NFFD in an approximate manner proposed by Tseng et al. (2000), and the same conclusions have been drawn as those presented here for the time-domain approach. For space-saving reasons, we refrain from plotting the frequency-domain characteristics, the more so as we now introduce an effective, time-varying, FD-approximating filter.

\section{Adaptive FFD}

It can be concluded from our analysis and Fig. 1 that NFFD proves to have the excellent steady-state behavior 
at the cost of possible remarkable modeling errors in the transient state, as compared with FD. On the other hand, poor steady-state properties of FFD are (at least partly) compensated by the excellent behavior in the initial transient state. In fact, for $t \leq \bar{J}$, FFD is identical with FD. Now, we propose to combine FFD with NFFD so that the advantages of the two could be retained in the combined FFD-NFFD model.

Firstly, the combination should employ FFD until $t=\bar{J}$. Secondly, for $t=\bar{J}+1$, FFD should not be directly switched to NFFD as the two are still much different from each other at that time, in general. A sort of a "bumpless transfer" between FFD and NFFD should be rather provided. To this end, we propose to use a filter incorporating the exponential forgetting factor $\lambda$, with $0<\lambda<1$, which is a classical memory-managing measure often employed in adaptive estimation schemes. Accordingly, we employ the following, time-varying normalizing factor during the FFD-NFFD transfer:

$$
N(t)=N-(N-1) \lambda^{t-\bar{J}}, \quad t=\bar{J}+1, \ldots,
$$

where $N=N(\bar{J})$ is the normalizing factor selected according to Lemma 1 and computed as in Eqns. (15) and (16).

Obviously, the weight $w(t)=\lambda^{t-\bar{J}}$ can also be calculated recursively,

$$
w(t)=\lambda w(t-1), \quad t=\bar{J}+1, \ldots,
$$

with $w(\bar{J})=1$.

In our numerous simulation experiments, we have found that, typically, a "good" value of $\lambda$ should range from 0.95 to 0.999 . An optimal $\lambda$ depends on $\alpha$ and $\bar{J}$ and could be obtained from, e.g., an (off-line) LS minimization procedure, but this would involve some computational effort.

Summing up, we introduce an adaptive (normalized) FFD, for the calculation of Eqn. (6) in the form of

$$
\begin{array}{r}
\Delta_{N}^{\alpha} x(t, J)=x(t)+\frac{1}{N(t)} \sum_{j=1}^{J} P_{j}(\alpha) x(t) q^{-j}, \\
t=0,1, \ldots,
\end{array}
$$

with the following time-varying normalizing factor:

$$
N(t)= \begin{cases}1, & t \leq \bar{J} \quad(F F D), \\ N-(N-1) w(t), & t=\bar{J}+1, \ldots,\end{cases}
$$

where $N=N(\bar{J})$ is selected according to Lemma 1 and computed as in Eqns. (15) and (16), $w(t)$ is calculated as in Eqn. (33) and $\lambda$ is the exponential forgetting factor.

Note that for $t=\bar{J}$ the second equation in 35 would reduce to the first one (bumpless transfer), whereas, as $t \rightarrow \infty$, the second equation in 35 converges to $N=N(\bar{J})$, yielding NFFD and implying that the NFFD-related result of Theorem 4 is still valid for the AFFD-based system.

A very accurate and robust modeling performance of AFFD will be demonstrated in simulations.

\section{Perfect FFD}

Now that the advantageous effect of a time-varying normalizing factor $N(t)$ on FD modeling accuracy via NFFD has been indicated, we would like to select $N(t)$ so as to minimize a modeling error. Surprisingly, it will be shown that, under some circumstances, perfect modeling of FD with NFFD (with a time-varying normalizing factor $N(t)$ ) is achievable in that the infimum modeling error is possible, that is, zero. We will call such an NFFD a perfect (normalized) FFD.

Definition 3. Consider an NFFD with a time-varying modeling factor $N(t)$ as in Eqn. 34 and define its step signal modeling error with respect to FD as

$$
\epsilon_{N(t)}(t)=\left.\left(\Delta_{N(t)}^{\alpha} x(t, J)-\Delta^{\alpha} x(t)\right)\right|_{x(t)=x_{0} \mathbf{1}(t)} .
$$

Then perfect FFD is defined as an NFFD that provides $\epsilon_{N(t)}(t)=0 \forall t=0,1, \ldots$

Theorem 5. Consider an NFFD desribed by Eqn. (34), with a time-varying normalizing factor $N(t)$. Then PFFD is obtained if $N(t)$ is selected as

$$
N(t)=\left\{\begin{array}{l}
1 \text { for } t=1, \ldots, \bar{J} \\
\frac{1}{1+P_{\bar{J}+1}(\alpha) / N} \text { for } t=\bar{J}+1 \\
\frac{1}{\frac{1}{N(t-1)}+\left(\frac{1}{N(t-1)}-\frac{1}{N(t-2)}\right)\left(1-\frac{\alpha-1}{t}\right)} \\
\text { for } t=\bar{J}+2, \ldots
\end{array}\right.
$$

with $N=N(\bar{J})$ selected as in Lemma 1 and computed as in Eqns. (15) and (16), and $P_{\bar{J}+1}(\alpha)$ computed as in Eqn. (15).

Proof. See Appendix C.

Resulting from Theorem 5 as well as Lemma 1 and Remark 1 is the following steady-state result.

Corollary 1. Let $N=N(\bar{J})$ be selected as in Eqn. (7) and $N(t)$ be selected as in Eqn. (37). Then

$$
\lim _{t \rightarrow \infty} N(t)=N
$$

Clearly, as $t \rightarrow \infty$, PFFD approaches NFFD. 
Remark 11. We have introduced the term 'perfect' NFFD in analogy to perfect regulation/control/signal reconstruction problems, where 'ideal' modeling/control/reconstruction abilities are, like here, apparently related to the solution of the inverse problem (Latawiec, 2004; Hunek and Latawiec, 2011).

It is well known that 'perfect' solutions may lack robustness in that they may be sensitive to disturbances or specific properties of systems/signals under control/modeling, e.g., time-varying conditions.

Note that the 'perfect' solution to the NFFD modeling problem is in fact 'ideal' in that PFFD $\equiv$ FD, for the step signal $x(t)=x_{0} \mathbf{1}(t)$ only. This does not have to necessarily mean that the zero-error modeling accuracy will be retained for other forms of the signal $x(t)$.

It is finally interesting to note that, in spite of the finite-memory NFFD origin, both PFFD and AFFD constitute infinite-memory time-varying approximations to FD.

\section{Variable-order AFFD and PFFD}

8.1. Variable-order AFFD. The time-varying nature of AFFD results in the fact that only minor modifications are necessary to account for variable-order $\alpha=\alpha_{t}$. In fact, variable-order AFFD can be computed as in Eqns. (33) and (35), with $N=N_{t}(J)$ calculated as in Eqns. (21) and (22). Note that for the FFD component of AFFD, that is, for $N(t)=1$, we employ $P_{j}\left(\alpha_{t}\right)$, $j=1, \ldots, \bar{J}$.

8.2. Variable-order PFFD. Unfortunately, nothing like in Section 8.1 can be applied for PFFD. In fact, PFFD is the recurrence which cannot be adopted for time-varying $\alpha_{t}$. Clearly, Eqns. (C2)-C6 of Appendix $\mathrm{C}$ are not valid for the case of $\alpha=\alpha_{t}$. We have tried to construct some approximations to Eqn. (C2), but we have not succeeded to find one(s) that could lead to something competitive to variable-order AFFD. Now, variable-order PFFD is left for possible future research, with our preliminary pessimistic conclusions resulting from a number of our 'trial-and-simulations'.

\section{Computational effort revisited: Part 2}

Time complexity $\mathcal{T}_{A F F D}(\bar{J})$ for one step of the simulation process for adaptive NFFD with $\alpha=$ const and $N(t)$ calculated as in (32) is

$$
\mathcal{T}_{\mathrm{AFFD}}(\bar{J})=2 \bar{J}+10 \in \mathcal{O}(\bar{J})
$$

and for perfect NFFD it is

$$
\mathcal{T}_{\mathrm{PFFD}}(\bar{J})=2 \bar{J}+15 \in \mathcal{O}(\bar{J}) .
$$

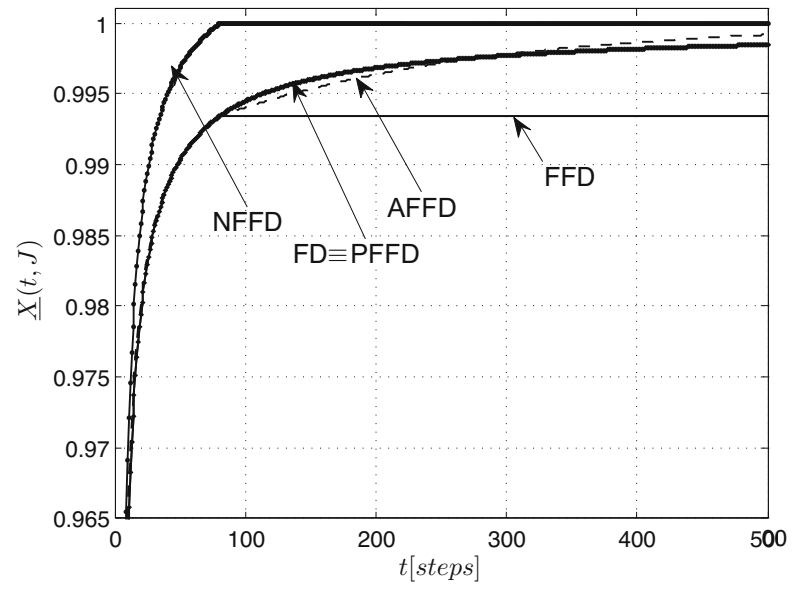

Fig. 4. Time plots of $\underline{X}(t, J)$ for FD/FFD/NFFD/AFFD/PFFD models: Example 4

Time complexity $\mathcal{T}_{\text {AFFDvar }}(\bar{J})$ for one step of the simulation process for variable-order AFFD (with $\alpha=$ $\left.\alpha_{t}\right)$ is

$$
\mathcal{T}_{\text {AFFDvar }}(\bar{J})=6 \bar{J}+7 \in \mathcal{O}(\bar{J}) .
$$

Again, linear time algorithms are obtained.

\section{Simulation examples: Part 2}

Example 4. Consider FD/FFD/NFFD/AFFD/PFFD fractional differences with $N$ selected as in Lemma 1 and $\alpha=0.9, \bar{J}=80, \lambda=0.995$. Figure 4 presents zoomed time plots of $\underline{X}(t, J)=-X(t, J)$ for all the models, with $x(t)=\mathbf{1}(t)$. It can be seen from Fig. 4 that the best, 'ideal' performance is provided by PFFD, which is not surprising under the step signal.

Example 5. Consider FD/FFD/NFFD/AFFD/PFFD fractional models as in Example 4, with $\lambda=0.9985$ for the AFFD model. A time plot of the realization of a (stochastic) signal $x(t)$ is presented in Fig. 5. The mean square prediction errors (MSPEs) for the analyzed models are presented in Table 2 , and zoomed time plots of $\underline{X}(t, J)$ for all the differences are shown in Fig. 6. The highest

Table 2. MSPE for FFD/NFFD/AFFD/PFFD models
\begin{tabular}{|c||c|c|c|c|}
\hline Model & FFD & NFFD & AFFD & PFFD \\
\hline MSPE & 0.0211 & 0.0083 & 0.0030 & $\mathbf{0 . 0 0 0 2 8}$ \\
\hline
\end{tabular}

modeling accuracy can be observed here for the PFFD model and the modeling performance for the AFFD model is also high. Note that the MSPE represents the LS fit of the models considered with respect to FD.

Example 6. Consider the model specifications as in Example 5, but for $x(t)=t$. The MSPE and the normalized MSPE (NMSPE) with respect to FD for the 


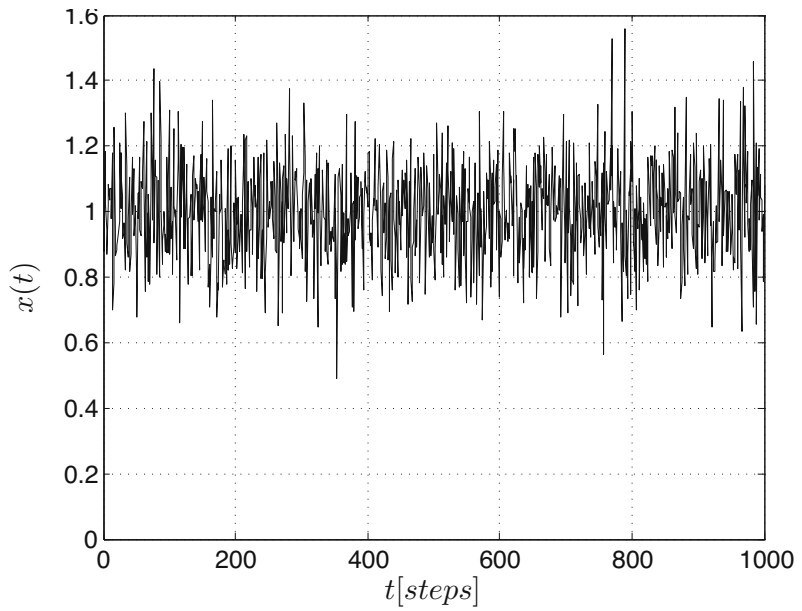

Fig. 5. Time plot of $x(t)$ for FD/FFD/NFFD/AFFD/PFFD models: Example 5

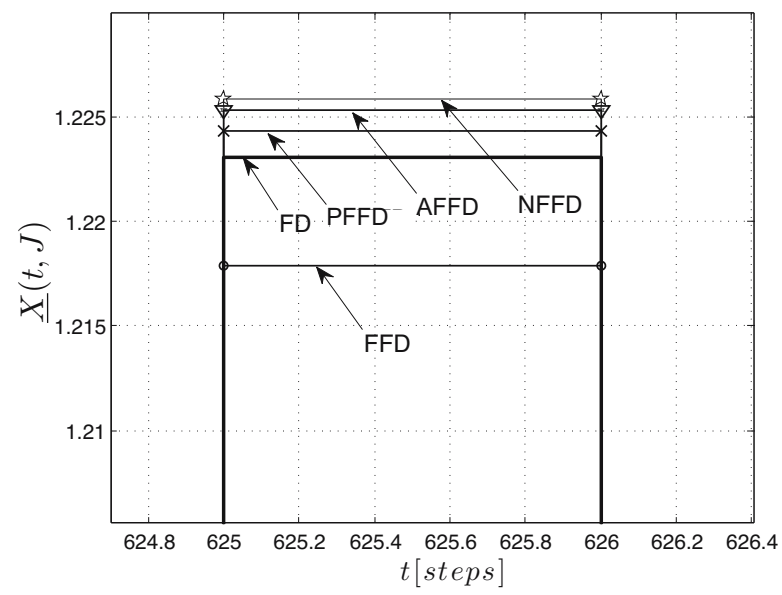

Fig. 6. Time plots of $\underline{X}(t, J)$ for FD/FFD/NFFD/AFFD/PFFD models (one step zoom): Example 5

analyzed models are presented in Table 3 and time plots of $\underline{X}(t, J)$ for the differences are shown in Fig. 7 Clearly,

Table 3. MSPE and NMSPE for FFD/NFFD/AFFD/PFFD mod-

\begin{tabular}{|c||c|c|c|c|}
\hline Model & FFD & NFFD & AFFD & PFFD \\
\hline MSPE & 4815 & 2640 & $\mathbf{4 3 . 8 3}$ & 842.2 \\
\hline NMSPE & $5.817 \mathrm{e}-5$ & $3.183 \mathrm{e}-5$ & $\mathbf{5 . 3 1 5 e - 7}$ & $1.016 \mathrm{e}-5$ \\
\hline
\end{tabular}

the best performance comes from the AFFD model now.

Example 7. Consider the AFFD model as in Example 4 and the signal $x(t)$ depicted in Fig. 5 (note how low $\bar{J}=80$ is here). The MSPE for AFFD with respect to FD for $\lambda=v a r$ is presented in Table 4. Selection of the forgetting factor $\lambda$ for AFFD is thus not that critical, with the best performance obtained here for $\lambda=0.995$.

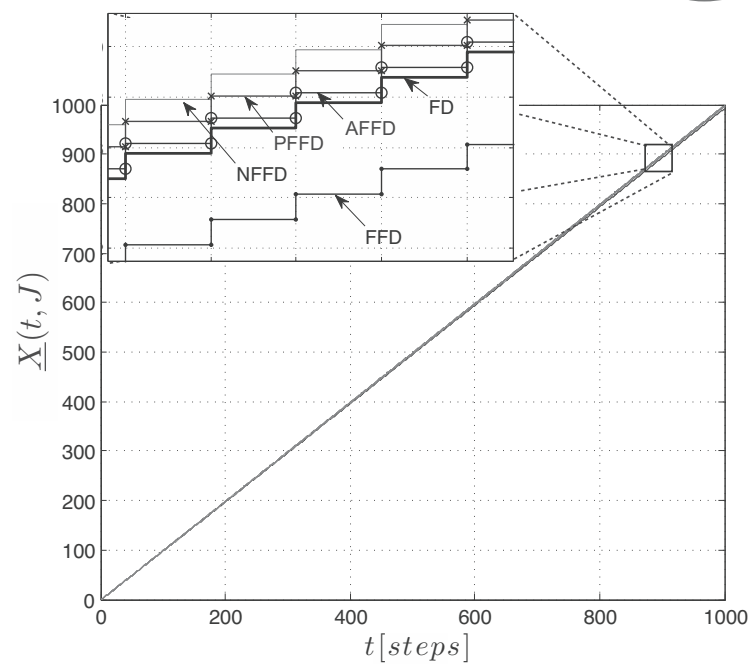

Fig. 7. Time plots of $\underline{X}(t, J)$ for FD/FFD/NFFD/AFFD/PFFD models: Example 6

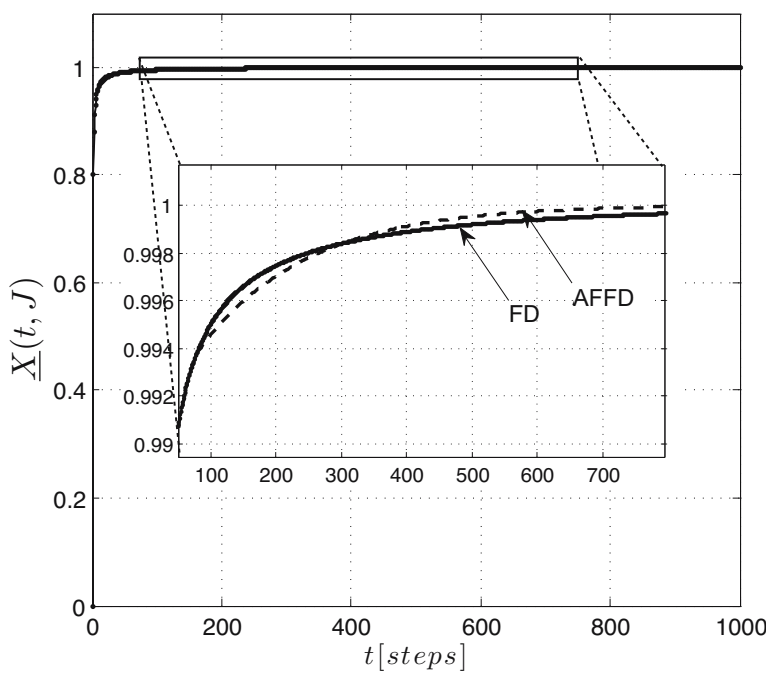

Fig. 8. Time plots of $\underline{X}(t, J)$ for FD/AFFD models: Example 8

Table 4. MSPE for the AFFD model with $\lambda=v a r$.

\begin{tabular}{|c||c|c|c|c|c|}
\hline$\lambda$ & 0.98 & 0.99 & 0.995 & 0.998 & 0.999 \\
\hline MSPE & $2.8 \mathrm{e}-3$ & $1.8 \mathrm{e}-3$ & $\mathbf{8 . 3 e}-4$ & $1.7 \mathrm{e}-3$ & $5.6 \mathrm{e}-3$ \\
\hline
\end{tabular}

Example 8. Consider a varying-order AFFD model as in Example $4 \alpha_{t}$ as in Example 3 and $\lambda=0.9985$. Time plots of $\underline{X}(t, J)$ for FD/AFFD are presented in Fig. 8, It is striking that even in this variable-order case the $\underline{X}(t, J)$ signals for FD and AFFD are hardly distinguishable. On the other hand, we could not modify PFFD to obtain comparable results.

Example 9. Consider FFD/NFFD/AFFD/PFFD-based state space models with the elements $A_{d}, B, C, D$ and $\alpha$ as in Example 1 the time-varying $\alpha_{t}$ as in Example 6 
$\bar{J}=100$ and $\lambda=0.99$ for the AFFD model. The MSPE and the NMSPE of the models for the input signal as in Example 6 are presented in Table 5.

Table 5. MSPE and the NMSPE for FFD/NFFD/AFFD/PFFDbased state-space models.

\begin{tabular}{|c||c|c|c|c|}
\hline Model & FFD & NFFD & AFFD & PFFD \\
\hline MSPE & $7.73 e+7$ & $6.74 \mathrm{e}+7$ & $\mathbf{1 . 8 9 e}+\mathbf{6}$ & $2.21 \mathrm{e}+7$ \\
\hline NMSPE & $1.53 \mathrm{e}-3$ & $1.34 \mathrm{e}-3$ & $\mathbf{3 . 7 5 e - 5}$ & $4.38 \mathrm{e}-4$ \\
\hline
\end{tabular}

The results of the above simulation examples for Series 2 strongly support our theoretical achievements of Sections 6-8. In particular, our robustness-related reservations against (otherwise theoretically attractive) PFFD are confirmed for non-step signals and, in particular, for the variable-order case. Therefore, we strongly advocate the use of our robust AFFD in the modeling of discrete-time fractional-order systems.

\section{Conclusion}

This paper has offered a series of original results in the modeling of discrete-time fractional-difference systems using finite/infinite-memory approximations to FD, namely, finite FD and, in particular, normalized finite FD. The steady-state error analyses for FFD and NFFD resulted in the introduction of a specific "normalizing factor", thus providing steady-state error-free modeling of NFFD-based systems. The stability analysis for NFFD-based state space systems revealed the stability condition which includes those for FD and FFD-based systems. A recursive algorithm for computation of NFFD yielded essential computational savings as compared to its off-line version.

Our main achievement is the presentation of various normalizing factors for NFFD, including time-varying ones, whose crucial influence on the quality of FD approximations was demonstrated both analytically and simulation-wise. In particular, an introduced adaptive version of NFFD (AFFD), being a new infinite-memory approximation to FD, essentially improved the modeling accuracy for NFFD in the transient state, even in the case of a variable fractional order. Also, we offered perfect FFD, providing an excellent performance of modeling of FD by means of NFFD, but only for constant signals (or constant-component ones) and a constant fractional order. We finally recommended robust AFFD as the most valuable tool for the modeling of FD, which was confirmed in numerous simulations.

Finally, we would like to stress the role of an $a$ priori knowledge in our FD modeling approach. Since the original mathematical and physical nature of FD is time-domain, we use this a priori knowledge to find a "good" FFD-based approximation to FD. In fact, an initial part of FFD is just FD so it "suffices" to accurately (and robustly) model the remaining part of FFD. Therefore, a consecution FD-FFD-NFFD-AFFD/PFFD is the natural one, just making use of the a priori knowledge which is FD and FFD. It should be emphasized that no such a priori knowledge has ever been used when modeling FD with any other filters (e.g., FIR, IIR, ARX-like, OBF), in particular, in the frequency domain. In fact, in the latter case a filter model of FD covers the whole frequency range, unnecessarily neglecting the a priori information that $\mathrm{FFD}=\mathrm{FD}$ in the high-frequency range. In other words, the FFD part of our AFFD approximation to FD covers the high-frequency range, the NFFD supplement fits the steady state whereas the medium-frequency range is modeled by the 'adaptive' part of AFFD. Well, with PFFD constituting a nice theoretical support of the whole FFD/NFFD involvement.

Our future research work will be concentrated on a rigorous frequency-domain analysis of AFFD, which is a real challenge in view of the specific structure of that nonlinear time-varying filter.

\section{Acknowledgment}

This article is an extended version of the paper presented at the 16th International Conference on Methods and Models in Automation and Robotics (MMAR), Międzyzdroje, Poland, 2011.

\section{References}

Bandrowski, B., Karczewska, A. and Rozmej, P. (2010). Numerical solutions to integral equations equivalent to differential equations with fractional time, International Journal of Applied Mathematics and Computer Science 20(2): 261-269, DOI: 10.2478/v10006-010-0019-1.

Barbosa, R. and Machado, J. (2006). Implementation of discrete-time fractional-order controllers based on LS approximations, Acta Polytechnica Hungarica 3(4): 5-22.

Busłowicz, M. and Kaczorek, T. (2009). Simple conditions for practical stability of positive fractional discrete-time linear systems, International Journal of Applied Mathematics and Computer Science 19(2): 263-269, DOI: 10.2478/v10006-009-0022-6.

Chen, Y., Vinagre, B. and Podlubny, I. (2003). A new discretization method for fractional order differentiators via continued fraction expansion, Proceedings of DETC'2003, ASME Design Engineering Technical Conferences, Chicago, IL, USA, Vol. 340, pp. 349-362.

Debeljković, D.L., Aleksendrić, M., Yi-Yong, N. and Zhang, Q. (2002). Lyapunov and nonlyapunov stability of linear discrete time delay systems, Facta Universitatis Mechanical Engineering 14(9-10): 1147-1160.

Delavari, H., Ranjbar, A., Ghaderi, R. and Momani, S. (2010), Fractional order control of a coupled tank, Nonlinear Dynamics 61(3): 383-397. 
Dzieliński, A. and Sierociuk, D. (2008). Stability of discrete fractional order state-space systems, Journal of Vibration and Control 14(9-10): 1543-1556.

Guermah, S., Djennoune, S. and Bettayeb, M. (2010). A new approach for stability analysis of linear discrete-time fractional-order systems, in D. Baleanu, Z.B. Güvenç and J.A.T. Machado (Eds.), New Trends in Nanotechnology and Fractional Calculus Applications, Springer, Dordrecht, pp. 151-162.

Hunek, W.P. and Latawiec, K.J. (2011). A study on new right/left inverses of nonsquare polynomial matrices, International Journal of Applied Mathematics and Computer Science 21(2): 331-348, DOI: 10.2478/v10006-011-0025-y.

Kaczorek, T. (2008). Practical stability of positive fractional discrete-time linear systems, Bulletin of the Polish Academy of Sciences: Technical Sciences 56(4): 313-317.

Latawiec, K.J. (2004). The Power of Inverse Systems in Linear and Nonlinear Modeling and Control, Opole University of Technology Press, Opole.

Liavas, A.P. and Regalia, P. (1999). On the numerical stability and accuracy of the conventional recursive least squares algorithm, IEEE Transactions on Signal Processing 47(1): $88-96$.

Lubich, C.H. (1986). Discretized fractional calculus, SIAM Journal on Mathematical Analysis 17(3): 704-719.

Maione, G. (2006). A digital, noninteger order, differentiator using laguerre orthogonal sequences, International Journal of Intelligent Control and Systems 11(2): 77-81.

Miller, K. and Ross, B. (1993). An Introduction to the Fractional Calculus and Fractional Differential Equations, Willey, New York, NY.

Momani, S. and Odibat, Z. (2007). Numerical approach to differential equations of fractional order, Journal of Computational and Applied Mathematics 207(1): 96 - 110.

Monje, C., Chen, Y., Vinagre, B., Xue, D. and Feliu, V. (2010). Fractional-order Systems and Controls, Springer-Verlag, London.

Oldham, K. and Spanier, J. (1974). The Fractional Calculus, Academic Press, Orlando, FL.

Ortigueira, M.D. (2000). Introduction to fractional linear systems, II: Discrete-time case, IEE Proceedings on Vision, Image and Signal Processing 147(1): 71-78.

Ostalczyk, P. (2000). The non-integer difference of the discrete-time function and its application to the control system synthesis, International Journal of Systems Science 31(12): 1551-1561.

Ostalczyk, P. (2010). Stability analysis of a discrete-time system with a variable-fractional-order controller, Bulletin of the Polish Academy of Sciences: Technical Sciences 58(4): 613-619.

Petráš, I., Dorčák, L. and Koštial, I. (2000). The modelling and analysis of fractional-order control systems in discrete domain, Proceedings of the International Carpatian Control Conference, High Tatras, Slovak Republic, pp. 257-260.
Petráš, I. and Vinagre, B. (2002). Practical application of digital fractional-order controller to temperature control, Acta Montanistica Slovaca 7(2): 131-137.

Podlubny, I. (1999). Fractional Differential Equations, Academic Press, Orlando, FL.

Riu, D., Retiére, N. and Ivanes, M. (2001). Turbine generator modeling by non-integer order systems, IEEE International Conference on Electric Machines and Drives, Cambridge, MA, USA, pp. 185-187.

Saeedi, H., Mollahasani, N., Moghadam, M.M. and Chuev, G.N. (2011). An operational Haar wavelet method for solving fractional Volterra integral equations, International Journal of Applied Mathematics and Computer Science 21(3): 535-547, DOI: 10.2478/v10006-011-0042-x.

Sierociuk, D. and Dzieliński, A. (2006). Fractional Kalman filter algorithm for states, parameters and order of fractional system estimation, International Journal of Applied Mathematics and Computer Science 16(1): 129-140.

Stanisławski, R. (2009). Identification of open-loop stable linear systems using fractional orthonormal basis functions, Proceedings of the 14th International Conference on Methods and Models in Automation and Robotics, Międzyzdroje, Poland, pp. 935-985.

Stanisławski, R. and Latawiec, K.J. (2010). Modeling of open-loop stable linear systems using a combination of a finite fractional derivative and orthonormal basis functions, Proceedings of the 15th International Conference on Methods and Models in Automation and Robotics, Międzyzdroje, Poland, pp. 411-414.

Stanisławski, R. and Latawiec, K.J. (2011). Finite approximations of a discrete-time fractional derivative, 16th International Conference on Methods and Models in Automation and Robotics, Międzyzdroje, Poland, pp. 142-145.

Stojanovic, S.B. and Debeljkovic, D.L. (2010). Simple stability conditions of linear discrete time systems with multiple delay, Serbian Journal of Electrical Engineering 7(1): 69-79.

Sun, H., Chen, W. and Chen, Y. (2009). Variable-order fractional differential operators in anomalous diffusion modeling, Physica A: Statistical Mechanics and Its Applications 388(21): 4586-4592.

Tseng, C., Pei, S. and Hsia, S. (2000). Computation of fractional derivatives using Fourier transform and digital FIR diferentiator, Signal Processing 80(1): 151-159.

Valério, D. and Sá da Costa, J. (2011). Variable-order fractional derivatives and their numerical approximations, Signal Processing 91(3): 470-483.

Verhaegen, M. H. (1989). Round-off error propagation in four generally-applicable, recursive, least-squares estimation schemes, Automatica 25(3): 437-444.

Vinagre, B., Podlubny, I., Hernandez, A. and Feliu, V. (2000). Some approximations of fractional order operators used in control theory and applications, Fractional Calculus \& Applied Analysis 3(3): 945-950. 
Zaborowsky, V. and Meylaov, R. (2001). Informational network traffic model based on fractional calculus, Proceedings of the International Conference on Info-tech and Info-net, ICII 2001, Beijing, China, Vol. 1, pp. 58-63.

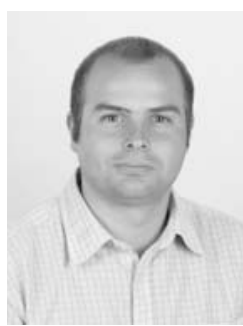

Rafał Stanisławski received his M.Sc. and Ph.D. degrees in electrical engineering from the Faculty of Electrical, Control and Computer Engineering, Opole University of Technology, Opole, Poland. $\mathrm{He}$ is an assistant professor at the Institute of Control and Computer Engineering. His research interests concentrate on modeling, identification and control of dynamic systems, including nonlinear and fractional systems and orthonormal basis functions.

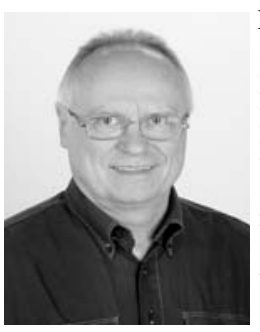

Krzysztof J. Latawiec received his habilitation degree in control and robotics in 1999. Currently he is a professor of control and robotics and the head of the Control and Electronics Group at the Faculty of Electrical, Control and Computer Engineering, Opole University of Technology. His research interests concentrate on system identification, multivariable control, adaptive and robust control (also in networks), and fractional systems.

\section{Appendix A}

\section{Proof of Theorem 3}

Proof. According to the arguments presented by Debeljković et al. (2002), a system described by the equation

$$
x(k+1)=A_{0} x(k)+\sum_{i=1}^{J} A_{i} x(k-1)
$$

is asymptotically stable if the following condition is satisfied:

$$
\sum_{i=0}^{J}\left\|A_{i}\right\|<1
$$

In our case,

$$
A_{i}= \begin{cases}A_{d}+\frac{\alpha}{N} I, & i=0, \\ -\frac{P_{j+1}(\alpha)}{N} I, & i \in(1, J-1),\end{cases}
$$

and so

$$
\sum_{i=0}^{J-1}\left\|A_{i}\right\|=\left\|A_{d}+\frac{\alpha}{N} I\right\|+\sum_{i=2}^{J}\left\|-\frac{1}{N} P_{j}(\alpha) I\right\| .
$$

Since for $0<\alpha<1$ we have $P_{i}(\alpha)<0, i=$ $1, \ldots, J$, we arrive at

$$
\begin{aligned}
\sum_{i=0}^{J-1}\left\|A_{i}\right\| & =\left\|A_{d}+\frac{\alpha}{N} I\right\|-\frac{1}{N} \sum_{i=2}^{J} P_{j}(\alpha) \\
& =\left\|A_{d}+\frac{\alpha}{N} I\right\|+1-\frac{\alpha}{N},
\end{aligned}
$$

with the second equality resulting from Lemma 1 Accounting for the inequality (A2), the result follows.

For $1<\alpha<2$, we have $P_{i}(\alpha)>0, i=2, \ldots, J$, and

$$
\begin{aligned}
\sum_{i=0}^{J-1}\left\|A_{i}\right\| & =\left\|A_{d}+\frac{\alpha}{N} I\right\|+\frac{1}{N} \sum_{i=2}^{J} P_{j}(\alpha) \\
& =\left\|A_{d}+\frac{\alpha}{N} I\right\|-1+\frac{\alpha}{N}
\end{aligned}
$$

which completes the proof.

\section{Appendix B}

\section{Proof of Theorem 4}

Proof. Denote by $x_{s s}^{n f d}$ and $x_{s s}^{f d}$ steady-state values of the state vectors for FFD/NFFD and FD, respectively. Then, by virtue of Eqn. 27], we have

$$
x_{s s}^{n f d}=\left(-A_{d}+F\right)^{-1} B u_{s s},
$$

where $F$ is given by Eqn. (31) and

$$
\begin{aligned}
x_{s s}^{f d} & =\left(-A_{d}+\left[1+\sum_{j=1}^{\infty} P_{j}(\alpha)\right] I\right)^{-1} B u_{s s} \\
& =-A_{d}^{-1} B u_{s s} .
\end{aligned}
$$

Applying the standard formula for the inverse of a sum of two matrices, Eqn. (B1) can be written in the form of

$$
x_{s s}^{n f d}=\left[-A_{d}^{-1}+\left(F-A_{d}\right)^{-1} F A_{d}^{-1}\right] B u_{s s} .
$$

Accounting for the output equation (25), we arrive at (30).

\section{Appendix C}

\section{Proof of Theorem 5}

Proof. For $x(t)=x_{0} \mathbf{1}(t)$, we need to have

$$
\left.\Delta_{N(t)}^{\alpha} x(t, J)=\Delta^{\alpha} x(t)\right)\left.\right|_{x(t)=x_{0} \mathbf{1}(t)}, \quad \forall t=0,1, \ldots,
$$

that is,

$$
\begin{array}{r}
x_{0}+\sum_{j=1}^{t} P_{j}(\alpha) x_{0}=x_{0}+\frac{1}{N(t)} \sum_{j=1}^{J} P_{j}(\alpha) x_{0}, \\
\forall t=1, \ldots
\end{array}
$$

Since $J=\min (t, \bar{J})=t$ for $t=1, \ldots, \bar{J}$, we immediately obtain $N(t)=1$ (FFD). 
For $t=\bar{J}+1$,

$$
\begin{aligned}
N(t) & =\frac{\sum_{j=1}^{J} P_{j}(\alpha)}{\sum_{j=1}^{t} P_{j}(\alpha)} \\
& =\frac{\sum_{j=1}^{\bar{J}} P_{j}(\alpha)}{\sum_{j=1}^{\bar{J}} P_{j}(\alpha)+P_{\bar{J}+1}(\alpha)} \\
& =\frac{N}{N+P_{\bar{J}+1}(\alpha)},
\end{aligned}
$$

where $N=N(\bar{J})$ is selected as in Lemma 1

For $t \geq \bar{J}+2$ we have

$$
\begin{aligned}
N(t) & =\frac{\sum_{j=1}^{J} P_{j}(\alpha)}{\sum_{j=1}^{t} P_{j}(\alpha)} \\
& =\frac{\sum_{j=1}^{\bar{J}} P_{j}(\alpha)}{\sum_{j=1}^{t-1} P_{j}(\alpha)+P_{t}(\alpha)} \\
& =\frac{N}{\frac{N}{N(t-1)}+P_{t}(\alpha)} .
\end{aligned}
$$

For $x(t)=x_{0} \mathbf{1}(t)$, from Eqn. (1) we have

$$
P_{t}(\alpha)=\frac{\Delta^{\alpha} x(t)-\Delta^{\alpha} x(t-1)}{x_{0}} .
$$

Since

$$
\begin{aligned}
P_{t}(\alpha) & =P_{t-1}(\alpha)\left(1-\frac{\alpha-1}{t}\right) \\
& =\left(\frac{\Delta^{\alpha} x(t-1)-\Delta^{\alpha} x(t-2)}{x_{0}}\right)\left(1-\frac{\alpha-1}{t}\right) \\
& =N \frac{N(t-2)-N(t-1)}{N(t-1) N(t-2)}\left(1-\frac{\alpha-1}{t}\right),
\end{aligned}
$$

we arrive at Eqn. (37).

Received: 16 June 2011

Revised: 18 November 2011

Re-revised: 16 April 2012 